\title{
24. Die Lebenssituationen von Personen in der zweiten Lebenshälfte mit und ohne Migrationshintergrund
}

\author{
Daniela Klaus \& Helen Baykara-Krumme
}

\section{Kernaussagen}

Die 40- bis 85-Jährigen mit Migrationshintergrund sind eine sehr heterogene Gruppe, die sich in unterschiedlichem Ausmaß von Personen ohne Migrationshintergrund unterscheiden: Zugewanderte aus den Ländern Asiens, Afrikas, Lateinamerikas, des Mittleren und Nahen Ostens und anderen nicht-westlichen Ländern außerhalb der EU sowie frühere Arbeitsmigrantinnen und -migranten und (Spät-)Aussiedlerinnen und -Aussiedler unterscheiden sich am stärksten von Personen ohne Migrationshintergrund. Hingegen sind Zugewanderte aus westlichen Ländern, der Europäischen Union sowie Angehörige der zweiten Generation denjenigen ohne Migrationshintergrund vergleichsweise ähnlich.

Personen mit Migrationshintergrund sind sozioökonomisch schlechter gestellt als Personen ohne Migrationshintergrund: Während die Armutsquote unter Personen ohne Migrationshintergrund bei 9,7 Prozent liegt, fällt sie unter den Arbeitsmigrantinnen und -migranten (37,1 Prozent) und den Zugewanderten aus den Ländern Asiens, Afrikas, Lateinamerikas, des Mittleren und Nahen Ostens und anderen nicht-westlichen Ländern außerhalb der EU (40,5 Prozent) deutlich höher aus.

Die vergleichsweise schlechte Gesundheit von Personen mit Migrationshintergrund zeigt sich vor allem bei den Arbeitsmigrantinnen und -migranten: Arbeitsmigrantinnen und -migranten weisen zu 38,1 Prozent funktionale Einschränkungen auf gegenüber 24,4 Prozent der Personen ohne Migrationshintergrund. Während 40,4 Prozent dieser Migrantengruppe von mindestens leichten depressiven Symptomen berichten, sind es nur 26,8 Prozent bei den Personen ohne Migrationshintergrund.

Ko-Residenz mit erwachsenen Kindern ist unter Arbeitsmigrantinnen und -migranten und Zugewanderten aus Afrika, Asien, Lateinamerika, dem Mittleren und Nahen Osten sowie weiteren Nicht-EU-Ländern am weitesten verbreitet: Arbeitsmigrantinnen und -migranten wohnen am häufigsten mit ihren erwachsenen Kindern zusammen (49,0 Prozent) - anders als Eltern ohne Migrationshintergrund (23,3 Prozent) und Angehörige der zweiten Generation (20,5 Prozent).

Migrantinnen und Migranten üben seltener ein Ehrenamt aus als Personen ohne Migrationshintergrund und Angehörige der zweiten Generation: Vor allem Arbeitsmigrantinnen und -migranten (3,3 Prozent) sind im Vergleich zu Personen ohne Migrationshintergrund (20,9 Prozent) und Angehörigen der zweiten Generation (16,7 Prozent) seltener ehrenamtlich engagiert. 


\subsection{Einleitung}

Knapp 15 Prozent der Personen in der zweiten Lebenshälfte (40- bis 85 -Jährige) haben einen Migrationshintergrund (Statistisches Bundesamt 2015). Eingewanderte und ihre Nachkommen sind insgesamt noch deutlich jünger als die Bevölkerung ohne Migrationshintergrund, doch bereits seit einigen Jahren erfahren sie eine "überproportionale demographische Alterung" (Zeman 2005: 23) ${ }^{1}$ - eine Entwicklung, die sich zukünftig fortsetzen wird, vor allem weil klassische Zuwanderergruppen der Nachkriegszeit ins Renten- und zunehmend auch ins hohe Alter kommen.

Die Forschung belegt eine anhaltende strukturelle Schlechterstellung von Personen mit Migrationshintergrund im Vergleich zur Bevölkerung ohne Migrationshintergrund, die sich im Alter in der Ausstattung mit finanziellen Ressourcen, in den Wohnverhältnissen und im Gesundheitszustand negativ niederschlägt (Baykara-Krumme, Motel-Klingebiel, \& Schimany 2012; BMFSFJ 2005; Schimany, Rühl, \& Kohls 2012; Schopf \& Naegele 2005; HeinrichBöll-Stiftung 2012). Allerdings ist bislang wenig darüber bekannt, welche Gruppen von älteren Personen mit Migrationshintergrund besonders benachteiligt sind. Das liegt vor allem daran, dass für die Beschreibung der Lebenssituation Älterer mit Migrationshintergrund bis in die 1990er Jahre lediglich kleinere, auf spezielle $\mathrm{Zu}$ wanderungsgruppen beschränkte Studien zur Verfügung standen (Baykara-Krumme \& Hoff 2006). Zudem wurde in der amtlichen Statistik (Melde- und Ausländerregister) wie auch im Mikrozensus lange Zeit nur zwischen Ausländern und Deutschen unterschieden. In den letzten Jahren hat sich die Datenlage etwas verbessert, insbesondere mit der Unterscheidung nach

1 Zu den Personen mit Migrationshintergrund gehören in Anlehnung an die Definition des Statistischen Bundesamtes alle Personen, die nach 1949 auf das heutige Gebiet der Bundesrepublik Deutschland zugewandert sind sowie alle in Deutschland Geborenen, die selbst eine ausländische Staatsangehörigkeit besitzen oder von denen mindestens ein Elternteil zugewandert ist oder eine ausländische Staatsangehörigkeit besitzt.
Migrationshintergrund infolge des 2005 in Kraft getretenen Zuwanderungsgesetzes. Doch nach wie vor sind einer differenzierten Beschreibung insofern Grenzen gesetzt, als einzelne Gruppen von Personen mit Migrationshintergrund in deutschlandweit repräsentativen Surveys wie dem Sozio-oekonomischen Panel (SOEP) oder dem Generations and Gender Survey (GGS) nur über wenige Fallzahlen vertreten sind. Das trifft umso stärker zu, wenn man nur für einen bestimmten Ausschnitt der Bevölkerung - wie ältere Personen - Aussagen treffen will. Am umfangreichsten wurden bislang die beiden klassischen Zuwanderergruppen in Deutschland untersucht: Arbeitsmigrantinnen und -migranten (und hier insbesondere Türkeistämmige) sowie (Spät-)Aussiedlerinnen und -Aussiedler.

(1) Die früheren Arbeitsmigrantinnen und -migranten und ihre Familien sind zwischen dem ersten Anwerbeabkommen mit Italien (1955) und dem Anwerbestopp (1973) beziehungsweise im Zuge des Familiennachzuges vor allem aus den Mittelmeerländern nach Deutschland gekommen. Zwar sind von den 9,6 Millionen zwischen 1955 und 1973 zugewanderten ausländischen Staatsangehörigen 5,7 Millionen wieder ausgereist (Schimany \& Baykara-Krumme 2012), doch viele sind geblieben und leben inzwischen durchschnittlich seit fast 45 Jahren in Deutschland. Viele von ihnen pendeln heute (saisonal) zwischen ihrem Herkunftsland und Deutschland. Laut Mikrozensus 2013 machen Personen aus diesen Anwerbeländern, die bis zum Anwerbestopp zugereist sind, in der Bevölkerung zwischen 40 und 85 Jahren mit Migrationshintergrund etwa 13 Prozent aus (Statistisches Bundesamt 2015).

(2) Die (Spät-)Aussiedlerinnen und-Aussiedler sind seit 1950 als deutsche Volkszugehörige aus Osteuropa (vor allem Polen und Rumänien) und verstärkt seit Mitte der 1980er infolge der politischen Umbrüche vor allem aus der ehemaligen Sowjetunion gekommen. Sie sind mit etwa 4,5 Millionen die größte Zuwanderungsgruppe in Deutschland (Vogel 2012) und leben im Mittel seit etwa 25 Jahren in Deutschland 
(Hoffmann \& Romeu Gordo 2016). Im Gegensatz zu den Arbeitsmigrantinnen und -migranten reisten sie von vornherein im Familienverband ein und mit der Absicht in Deutschland zu bleiben. Laut Mikrozensus 2013 machen sie unter den 40- bis 85-jährigen Personen mit Migrationshintergrund etwa 29 Prozent aus (Statistisches Bundesamt 2015).

Kaum erforscht ist die große Gruppe der übrigen Personen mit Migrationshintergrund, die sich als sehr heterogen und damit schwer fassbar darstellt. Diese Personen unterscheiden sich nach Herkunft, nach Wanderungserfahrung und -motiven sowie nach Einreisebedingungen. Basierend auf der Herkunftsregion und damit implizierten Merkmalen zur Ressourcenausstattung sowie (rechtlichen) Aufenthalts- und Lebensbedingungen in Deutschland sind mindestens zwei weitere Gruppen von Zuwanderern zu unterscheiden:

(3) Zunächst gibt es die Gruppe der Migrantinnen und Migranten aus westlichen Ländern beziehungsweise Ländern der Europäischen Union $(E U)$, die weder als klassische Arbeitsmigrantinnen und -migranten noch als (Spät-)Aussiedlerinnen und -Aussiedler nach Deutschland gekommen sind. Der Großteil der Zuzüge der letzten Jahre erfolgte aus Ländern, die im Jahr 2014 Teil der EU sind (Thum, Delkic, Kemnitz, Kluge, Marquardt, Motzek, Nagl, \& Zwerschke 2015). Zuwandererinnen und Zuwanderer aus der EU, dem europäischen Wirtschaftsraum (EWR) und der Schweiz sind ab 2004 vermehrt im Rahmen des Freizügigkeitsgesetzes nach Deutschland gekommen. Aufgrund ihrer Herkunft dürfte ihnen, wie auch den Zugewanderten aus anderen westlichen Industriestaaten (wie USA oder Australien) eine vergleichsweise gute sozioökonomische Ausstattung gemein sein sowie ein Aufenthaltsstatus, der eine gute Eingliederung in den deutschen Arbeitsmarkt ermöglicht.

(4) Es verbleiben Migrantinnen und Migranten aus Ländern bislang unberücksichtigter Regionen (Asien, Afrika, Lateinamerika, Naher und Mittlerer Osten) sowie aus Ländern Europas, die nicht dem Freizügigkeitsgesetz unterliegen. Mit Blick auf Aufenthaltsstatus, kulturelle Herkunft und Ressourcenausstattung ist für diese Gruppe von einer vergleichsweise großen Heterogenität auszugehen. Das betrifft ebenso ihre Migrationsmotivation beziehungsweise rechtlichen Möglichkeiten der Einwanderung aus diesen sogenannten Drittländern (Thum et al. 2015): Es ist anzunehmen, dass nur ein geringer Prozentsatz zu Erwerbs- oder Studienzwecken eingereist ist, während der Großteil als nachgezogene Familienangehörige oder Flüchtlinge nach Deutschland gekommen ist.

(5) Aufgrund Deutschlands relativ junger Einwanderungsgeschichte ist der Großteil der Menschen mit Migrationshintergrund in der zweiten Lebenshälfte noch selbst zugewandert. Von ihnen zu unterscheiden sind die Nachkommen aus Einwandererfamilien. In der migrationssoziologischen Forschung werden sie üblicherweise als zweite Generation bezeichnet und umfassen diejenigen, die als Kinder von Zugewanderten in Deutschland geboren wurden oder im frühen Kindesalter mit ihren Eltern eingereist sind. Charakteristisch für sie ist, dass sie vollständig oder größtenteils in Deutschland sozialisiert wurden. Angehörige dieser Gruppe erreichen inzwischen zunehmend ihre zweite Lebenshälfte.

Da sich die bisherige Forschung vor allem Arbeitsmigrantinnen und -migranten und (Spät-)Aussiedlerinnen und -Aussiedlern gewidmet hat, ist über die Lebensumstände älterer und alter Personen aus diesen beiden Gruppen am meisten bekannt. Dabei wurden im Wesentlichen drei große Dimensionen betrachtet: sozioökonomische Ausstattung, Gesundheit sowie soziale Einbettung und Partizipation.

Sozioökonomische Ausstattung: Im Jahr 2005 schloss der Sechste Bericht über die Lage der Ausländerinnen und Ausländer in Deutschland (Deutscher Bundestag 2005) unter anderem mit der Prognose, dass ältere Migrantinnen und Migranten diejenigen sind, die in Zukunft am stärksten von Armut betroffen sein werden. Bereits zu dieser Zeit gab es Hinweise für eine finanzielle und materielle Schlechterstellung von älteren Ausländerinnen und Ausländern, an deren benachteiligter Situation sich bis heute wenig geändert hat: Im Vergleich zur Bevölkerung ohne Migrationshintergrund desselben Alters beziehen sie geringere Einkommen, leben häu- 
figer von sozialstaatlichen Transferzahlungen, verfügen über kleinere Vermögen, weisen eine höhere Armutsquote auf, verfügen seltener über Wohneigentum und über weniger Wohnraum pro Person (Baykara-Krumme \& Hoff 2006; Menning \& Hoffmann 2009; Mika \& Tucci 2006; Özcan \& Seifert 2006; Tucci 2012; Tucci \& Yıldız 2012). In der Folge sind sie deutlich unzufriedener mit ihrer finanziellen Lage beziehungsweise Absicherung (Hubert, Althammer, \& Korucu-Rieger 2009; Özcan \& Seifert 2006) und haben ein niedrigeres subjektives Wohlbefinden (Baykara-Krumme \& Hoff 2006).

Zentrale Ursachen hierfür sind die geringe berufliche Qualifikation beziehungsweise die Nichtanerkennung ausländischer Abschlüsse, die niedrige berufliche Position und - daran anknüpfend - das geringe Lohnniveau sowie nachteilige Erwerbsbiografien. Dabei bestehen deutliche Unterschiede zwischen den Migrantengruppen, die sich letztlich in einer starken Heterogenität der Wirtschaftslage im Rentenalter niederschlagen (Frick, Grabka, GrohSamberg, Hertel, Tucci, \& Fries 2009; Romeu Gordo 2012; Tucci 2012). So haben Arbeitsmigrantinnen und -migranten aus der Türkei und dem ehemaligem Jugoslawien die niedrigsten Renteneinkommen, weil sie überwiegend als ungelernte Arbeitskräfte für den industriellen Sektor angeworben wurden und dort später im Zuge der De-Industrialisierung am stärksten vom Arbeitsplatzabbau betroffen waren. Neben einem relativ späten Beginn der Einzahlungen in die Rentenversicherung führten die aufgrund der unterprivilegierten Berufsposition unterdurchschnittlichen Beitragszahlungen $\mathrm{zu}$ den niedrigen Altersrenten. Dagegen haben (Spät-) Aussiedlerinnen und -Aussiedler höhere Ausbildungsabschlüsse, wenngleich sie aufgrund fehlender Anerkennung häufig eine berufliche Deklassierung auf dem deutschen Arbeitsmarkt erfahren haben (Greif, Grediga, \& Janikowski 2003). Doch sie erhielten bei ihrer Einwanderung umgehend die deutsche Staatsbürgerschaft, und diverse Eingliederungsprogramme wie Sprachkurse erhöhten ihre Chancen auf dem Arbeitsmarkt. Vor allem erlaubt ihnen das Fremdrentengesetz, im Heimatland erworbene Rentenansprüche in Deutschland geltend zu machen - bis 1992 in vollem Umfang und unter Einschluss mitziehender Angehöriger (Baumann \& Mika 2012). Offenbar verfügen sie auch häufiger über Wohneigentum in Deutschland als Arbeitsmigrantinnen und -migranten, nicht zuletzt weil sie häufiger in ländlichen Regionen mit einem günstigeren Wohnungsmarkt wohnen.

Gesundheit: Vergleichende Mortalitätsraten haben wiederholt gezeigt, dass Migrantinnen und Migranten im Allgemeinen eine geringere Sterblichkeit aufweisen als die einheimische Bevölkerung. Diese als ,Healthy-migrant Effekt bezeichnete Selektion wird dadurch hervorgerufen, dass typischerweise die Gesündesten ihr Heimatland verlassen (Höhne \& Schubert 2007). Allerdings hebt sich dieser Vorteil mit zunehmender Aufenthaltsdauer in Deutschland und zunehmendem Alter auf beziehungsweise kehrt sich sogar für bestimmte Migrantengruppen um (Kohls 2012; Menning \& Hoffmann 2009): Vor allem alte Arbeitsmigrantinnen und -migranten weisen ein hohes Sterberisiko auf. Das deutet sich bereits in hohen Raten von Frühverrentung und Erwerbsminderung unter den türkeistämmigen Arbeitsmigranten infolge körperlich anstrengender Arbeiten im industriellen Sektor an (Olbermann 2012).

Es ist belegt, dass ältere Personen mit Migrationshintergrund insgesamt häufiger und früher von gesundheitlichen Beeinträchtigungen betroffen sind als Personen ohne Migrationshintergrund - das gilt sowohl für physische wie auch psychische Erkrankungen und schlägt sich letztlich in einer schlechteren subjektiven Bewertung des eigenen Gesundheitszustandes nieder (Sahyazici \& Huxhold 2012; Olbermann 2012; Schopf \& Naegele 2005). Diese Schlechterstellung muss - dem Lifecourse Epidemiology Model (Spallek \& Razum 2008) folgend - konzeptionell als Resultat der Situation im Herkunftsland, des Migrationsereignisses und der Bedingungen im Aufnahmeland betrachtet werden. $\mathrm{Zu}$ vergleichsweise schlechter Gesundheit im Alter tragen so neben möglichen herkunftsbedingten Nachteilen ein ungünstigeres $\mathrm{Ge}$ sundheitsverhalten (BMFSFJ 2005), diskriminierende Erfahrungen auf dem deutschen Arbeits- und Wohnungsmarkt, schlechter Zugang 
$\mathrm{zu}$ sozioökonomische Ressourcen und (unter anderem sprachlich bedingte) Zugangsbarrieren zu Gesundheitsleistungen wie Prävention und Rehabilitation (Brzoska \& Razum 2015) bei. Vor allem der Zusammenhang zwischen sozioökonomischem Status und Gesundheit ist teilweise so stark (Kunst, Bos, Lahelma, Bartley, Lissau, Regidor, Mielck, Cardano, Dalstra, Geurts, Helmert, Lennartsson, Ramm, Spadea, Stronegger, \& Mackenbach 2005), dass Gruppenunterschiede allein hierdurch erklärt werden können (Sahyazici \& Huxhold 2012). Vor allem Migrantinnen und Migranten der ersten Generation weisen im Alter Gesundheitsdefizite auf, und hier vor allem die klassischen Gastarbeiterinnen und Gastarbeiter als Folge ihrer schlechteren Arbeits- und Lebensbedingungen.

Soziale Einbindung und Partizipation: Trotz sozioökonomischer und gesundheitlicher Benachteiligung sind ältere Personen mit Migrationshintergrund gut in soziale und vor allem familiale Netzwerke eingebunden - zum Teil sogar stärker als Personen gleichen Alters ohne Migrationshintergrund (Baykara-Krumme 2008b, 2008a; Baykara-Krumme \& Hoff 2006; Carnein \& Baykara-Krumme 2013; DietzelPapakyriakou 2005; Dietzel-Papakyriakou \& Olbermann 1996; Hubert et al. 2009; Vogel 2012; Steinbach 2013). Das liegt auch darin begründet, dass viele Migrantinnen und Migranten aus stärker familialistisch und kollektivistisch geprägten Kulturkreisen stammen, die familialen Zusammenhalt sehr viel stärker betonen als die deutsche Bevölkerung - nicht zuletzt auch aus ökonomischer Notwendigkeit heraus. Dieser Unterschied kann sich infolge der Migrationserfahrung verstärken, um mithilfe intensivierter Generationenbeziehungen in der neuen Umgebung besser zurecht zu kommen (Baykara-Krumme, Klaus, \& Steinbach 2011). Familien- oder Kettenmigration - die vor allem für die beiden größten Zuwanderergruppen charakteristisch sind - sind hierbei hilfreich.

Ältere Personen mit Migrationshintergrund leben im Durchschnitt seltener allein, in größeren Haushalten und häufiger zusammen mit erwachsenen Kindern als Personen ohne Migrationshintergrund. Sie unterhalten enge Familien- und Verwandtschaftsbeziehungen - oft auch dann, wenn eine Generation weit entfernt oder gar im Ausland lebt. Für die in der Literatur diskutierte Konfliktthese zwischen den Generationen (Baykara-Krumme et al. 2011) - begründet durch die räumliche Distanz infolge der Migration und ungleicher familienbezogener Erwartungen und Einstellungen - gibt es bislang kaum Hinweise. Vielmehr zeichnen sich die Beziehungen zwischen den Generationen durch ein hohes Unterstützungspotenzial aus und weisen ähnliche Transfermuster auf (vgl. Kapitel 16) wie die Generationenbeziehungen in Familien ohne Migrationshintergrund. Allerdings nimmt die mittlere Generation häufiger die Rolle von finanziellen Unterstützungsleistern in beide Richtungen ein - an Eltern (im Ausland) und erwachsene Kinder (Baykara-Krumme \& Hoff 2006) und zwischen verschiedenen Migrantengruppen besteht eine durchaus große Heterogenität (Baykara-Krumme 2008b).

Für Migrantinnen und Migranten wird seltener von freiwilligem Engagement und Mitgliedschaften in Vereinen und Organisationen berichtet als für ältere Personen ohne Migrationshintergrund (Baykara-Krumme \& Hoff 2006; Halm \& Sauer 2007). Gleichzeitig aber engagieren sich ältere (vor allem türkeistämmige) Zuwandererinnen und Zuwanderer durchaus stark in religiösen Gruppen, Migrantenvereinen und ethnisch organisierten Selbsthilfenetzwerken, die Bedürfnisse nach Geselligkeit, Informationsaustausch und gegenseitiger Unterstützung befriedigen (Dietzel-Papakyriakou 2005; Halm \& Sauer 2007; Huth 2012). Es tragen also sowohl die Familie als auch darüber hinaus gehende Zusammenschlüsse zur sozialen Einbettung der Älteren mit Migrationshintergrund bei und gewährleisten benötigte Hilfe. Dabei handelt es sich um weitgehend herkunftshomogene Gruppen, was im Alter einerseits durchaus positiv gesehen wird. Andererseits werden dadurch möglicherweise nicht alle Bedarfe abgedeckt.

Fragestellungen: In Anbetracht des bisherigen Forschungsstandes und basierend auf einem Konzept von Integration, das diese als „Mitgliedschaftswerdung und Angleichung der Lebensverhältnisse“ (Heckmann 2015: 82) begreift und im Ergebnis eine gleichberechtigte gesellschaftliche Mitgliedschaft impliziert, bei der 
ethnische Herkunft und Migrationshintergrund für die Ressourcenverteilung und die Strukturierung sozialer Beziehungen keine Rolle mehr spielen, lautet die leitende Fragestellung dieses Kapitels: Wie steht es um die gesellschaftliche Teilhabe der Älteren ohne Migrationshintergrund im Vergleich zu quantitativ und inhaltlich relevanten Gruppen von Personen mit Migrationshintergrund?

Diese Frage soll entlang der drei vorgestellten Dimensionen beantwortet werden. Allgemein wird angenommen, dass Ältere mit Migrationshintergrund gegenüber Personen gleichen Alters ohne Migrationshintergrund sozioökonomisch und gesundheitlich benachteiligt sind. Kaum Unterschiede sollten sich bezüglich der sozialen Einbindung und Partizipation zeigen.

Anhand einer differenzierten Betrachtung unterschiedlicher Gruppen von Personen mit Migrationshintergrund soll gezeigt werden, welche Migrantengruppen besonders benachteiligt sind. Begründet durch eine vergleichbare Ausstattung mit sozioökonomischen Ressourcen und ähnlichem rechtlichen Status, wird vermutet, dass sich Migrantinnen und Migranten aus westlichen Industrienationen und Ländern der EU sowie Angehörige der zweiten Generation in ähnlichen Lebenssituationen befinden wie Personen ohne Migrationshintergrund. Unterschiede zwischen Personen mit und ohne Mig-

\subsection{Datengrundlage ${ }^{2}$}

Daten. In der ersten Erhebung des Deutschen Alterssurveys (DEAS) im Jahr 1996 wurden nur Personen mit deutscher Staatsangehörigkeit befragt und im Jahr 2002 wurde eine eigenständige Ausländerstichprobe realisiert. Erst ab 2008 bildet die in Privathaushalten lebende Wohnbevölkerung Deutschlands - unabhängig von der Staatsangehörigkeit - die Grundgesamtheit für die Stichprobenziehung des DEAS (vgl. Kapitel

2 Die Daten des DEAS können für wissenschaftliche Zwecke kostenlos beim Forschungsdatenzentrum des DZA (www.fdz-dza.de) bezogen werden. rationshintergrund sollten vor allem auf größere Differenzen zu den früheren Arbeitsmigrantinnen und -migranten zurückzuführen sein, deren Zuwanderungs- und Aufenthaltsbedingungen in Deutschland vergleichsweise ungünstig waren.

Zusammenfassend formuliert geht es um folgende Fragen:

1. Sind Personen in der zweiten Lebenshälfte mit Migrationshintergrund nach wie vor gegenüber der Mehrheitsgesellschaft sozioökonomisch benachteiligt? Welche Personengruppen mit Migrationshintergrund sind hiervon besonders betroffen?

2. Weisen Personen mit Migrationshintergrund weiterhin eine schlechtere Gesundheit auf als Personen ohne Migrationshintergrund und gibt es bedeutsame Geschlechtsunterschiede und Unterschiede zwischen den Migrantengruppen?

3. Genießen Personen mit Migrationshintergrund weiterhin eine hohe familiale und soziale Einbindung oder gibt es Anzeichen für eine abnehmende Einbindung? Unterscheiden sich diesbezüglich die betrachteten Gruppen von Personen mit Migrationshintergrund?

2). Entsprechend sind in den Erhebungen 2008 und 2014 Personen mit Migrationshintergrund grundsätzlich Teil der Stichprobe. Auch erst in diesen beiden Erhebungsjahren werden ausreichend Informationen zur Feststellung des Migrationshintergrundes erhoben. Gleichwohl sind die Fallzahlen von Personen mit Migrationshintergrund in den einzelnen Erhebungen relativ gering. Entsprechend werden für die folgenden Analysen die Querschnittstichproben von 2008 und 2014 zusammengespielt. Auf diese Weise ist es erstmals möglich, die Lebenssituationen der Menschen mit Migrationshintergrund in 
der zweiten Lebenshälfte abzubilden. Befragt werden Personen zwischen 40 und 85 Jahren. Grundlage für alle hier verwendeten Indikatoren bilden die Daten, die im Rahmen eines mündlichen Interviews erhoben wurden.

Gruppen von Personen mit und ohne Migrationshintergrund. Zur Identifikation des Migrationshintergrundes erfragt der DEAS (1) den Geburtsort, (2) das Land, in dem die Kindheit und Jugend bis zum 16. Lebensjahr überwiegend verbracht wurde, (3) die aktuelle Staatsangehörigkeit, (4) das Jahr der Einbürgerung sowie (5) das Einreisejahr. Auf dieser Basis werden die Befragten einer der folgenden Gruppen zugeordnet:

Ehemalige Arbeitsmigrantinnen und -migranten sowie nachgezogene Ehepartnerinnen und -partner: In diese Gruppe fallen Befragte, die den Großteil ihrer Kindheit in den Anwerbeländern der Mittelmeerregion verbracht haben (vor allem Türkei, ehemaliges Jugoslawien, Griechenland, Italien, Spanien und Portugal). Hier werden nur Personen berücksichtigt, die zwischen dem ersten Abwerbeabkommen 1955 und dem 1979 weitgehend abgeschlossenen Familiennachzug (Meier-Braun 2002) nach Deutschland eingereist sind. Es zeigt sich, dass etwa ein Drittel (31,3 Prozent) von ihnen inzwischen eingebürgert ist. Weitere häufig vertretene (zweite) Staatsbürgerschaften entsprechen den bekannten Herkunftsländern (Italien/Griechenland: 24,9 Prozent; Türkei: 23,1 Prozent; Nachfolgestaaten des ehemaligen Jugoslawiens: 13,6 Prozent).

(Spät-)Aussiedlerinnen und -Aussiedler mit Ehepartnerinnen und -partnern: Als Angehörige dieser Gruppen gelten Befragte mit deutscher Staatsbürgerschaft, die aus früheren Ostgebieten stammen oder aus Ländern mit deutschstämmigen Bevölkerungsgruppen (ehemalige Sowjetunion, Polen, Rumänien, Ungarn) und die nach 1950 zugewandert sind. Hier werden nur Einwanderungen bis 2006 berücksichtigt, da die Zuwanderung von Spät-Aussiedlerinnen und Spät-Aussiedlern danach in keinem nennenswerten Umfang mehr stattfand.

Migrantinnen und Migranten aus westlichen und EU-Ländern: Hier werden Personen zusammengefasst, die keiner der beiden obigen Grup- pen angehören, aber den Großteil ihrer Kindheit in einem Land der Europäischen Union oder einem anderen westlichen Industrieland (Kanada, USA, Australien, Schweiz, Norwegen, Liechtenstein) verbracht haben. Ihre Zuordnung erfolgt unabhängig von der Staatsangehörigkeit, wobei sich zeigt, dass nur ein geringer Teil von ihnen eingebürgert ist (8,9 Prozent). Die große Mehrheit besitzt die Staatsbürgerschaft eines EULandes. Während 30,4 Prozent Staatsangehörige eines Landes sind, das erst nach 2004 der EU beigetreten ist, stammen 54,5 Prozent aus den langjährigen Mitgliedsstaaten der EU.

Sonstige Migrantinnen und Migranten: Angehörige dieser Gruppe sind keiner anderen bereits definierten Migrantengruppe zugeordnet. Diese Gruppe ist hinsichtlich der regionalen Herkunft ihrer Mitglieder, ihrem kulturellen Hintergrund sowie ihrer Ressourcenausstattung sehr heterogen zusammengesetzt. Hier finden sich Zugewanderte, die aus verschiedenen Ländern Afrikas, Asiens, Lateinamerikas, des Nahen oder Mittleren Ostens stammen oder ihre Kindheit in einem europäischen Land verbracht haben, das weder zur EU noch zum EWR gehört. Viele von ihnen sind bereits eingebürgert (36,0 Prozent). Weitere häufig vertretene Staatsbürgerschaften verweisen auf typische Herkunftsregionen: Türkei (18,7 Prozent), Nachfolgestaaten des ehemaligen Jugoslawiens (11,7 Prozent) und der ehemaligen Sowjetunion (14,1 Prozent). Zugewanderte aus diesen Ländern sind weder als klassische Arbeitsmigrantinnen und -migranten nach Deutschland gekommen noch im Rahmen der (Spät-)Aussiedlereinwanderung. Weitere 20,4 Prozent besitzen die Staatsangehörigkeit eines nicht-westlichen Landes außerhalb der EU-Grenzen.

Zweite Generation: Zu dieser Gruppe zählen Nachkommen von Zugewanderten, die entweder in Deutschland geboren wurden oder die im Ausland geboren wurden, aber den Großteil ihrer Kindheit in Deutschland verbracht haben. Da erst ab der Befragung im Jahr 2014 Informationen zur Herkunft der Eltern erfasst werden, können in Deutschland geborene Nachkommen von Zugewanderten nur dann als solche identifiziert werden, wenn sie zum Befragungszeitpunkt (noch) keine deutsche Staatsbürgerschaft 
besitzen oder erst nach ihrer Geburt eingebürgert wurden. ${ }^{3}$ Auch hier ist eine große Heterogenität der Angehörigen dieser Gruppe anzunehmen, die sich allerdings insofern ähneln, als sie (fast) ihr gesamtes Leben in Deutschland verbracht haben. Es zeigt sich entsprechend, dass die Mehrheit die deutsche Staatsangehörigkeit besitzt (73,3 Prozent). Weitere Staatsangehörigkeiten verweisen vor allem auf eine türkische $(6,7$ Prozent) und westeuropäische Herkunft (15,1 Prozent).

Als Personen ohne Migrationshintergrund gelten Befragte, die auf dem Gebiet des heutigen Deutschlands geboren wurden und seit ihrer Geburt die deutsche Staatsbürgerschaft besitzen oder vor 1950 zugezogen sind.

3 Personen, die als Kinder eingebürgerter Migrantinnen und Migranten bereits von Geburt an die deutsche Staatsbürgerschaft haben, können aufgrund fehlender Informationen nicht identifiziert werden. Sie werden fälschlicherweise den Personen ohne Migrationshintergrund zugeordnet. In den hier betrachteten $\mathrm{Ge}$ burtskohorten dürfte dieser Fehler jedoch sehr klein ausfallen.
In Tabelle 24-1 sind die Befragtenzahlen pro Gruppe sowie die gewichteten Stichprobenanteile zusammengestellt - getrennt für 2008 und 2014. Personen mit Migrationshintergrund machen jeweils etwa zehn Prozent der Stichprobe aus, was eine Unterschätzung gegenüber ihrem Anteil in der Gesamtbevölkerung der entsprechenden Altersgruppe bedeutet, der 15 Prozent beträgt (vgl. Kapitel 2). Etwa ein Prozent der Personen kann aufgrund fehlender oder widersprüchlicher Angaben keiner der inhaltlich definierten Gruppen zugeordnet werden. Diese Fälle bleiben in den späteren Analysen unberücksichtigt. (Spät-)Aussiedlerinnen und -Aussiedler sowie Angehörige der zweiten Generation bilden die stärksten Gruppen unter denjenigen mit Migrationshintergrund: Das ist durch ihren Anteil in der Bevölkerung dieses Alters begründet beziehungsweise verweist auf ihre vergleichsweise besseren Deutschkenntnisse, die bei der Durchführung der ausschließlich deutschsprachigen Interviews notwendig waren.

Tabelle 24-1: Verteilung von Personen mit und ohne Migrationshintergrund, in den Jahren 2008 und 2014 (absolut, in Prozent)

\begin{tabular}{lcccccc}
\hline & \multicolumn{2}{c}{2008} & \multicolumn{2}{c}{$\mathbf{2 0 1 4}$} & \multicolumn{2}{c}{ Gesamt } \\
& $\mathbf{N}$ & $\mathbf{\%}$ & $\mathbf{N}$ & $\%$ & $\mathbf{N}$ & $\%$ \\
\hline Ohne Migrationshintergrund & 5.676 & 90,2 & 5.508 & 90,0 & 11.184 & 90,1 \\
Mit Migrationshintergrund & & & & & & \\
$\quad$ ArbeitsmigrantInnen & 57 & 1,1 & 50 & 0,9 & 107 & 1,0 \\
(Spät-)AussiedlerInnen & 159 & 2,9 & 150 & 2,9 & 309 & 2,9 \\
MigrantInnen aus westlichen und EU-Ländern & 58 & 1,2 & 76 & 1,6 & 134 & 1,4 \\
Sonstige MigrantInnen & 73 & 1,6 & 77 & 1,8 & 150 & 1,7 \\
Zweite Generation & 129 & 2,0 & 120 & 2,4 & 249 & 2,2 \\
Unbekannt & 53 & 0,9 & 21 & 0,4 & 74 & 0,7 \\
Gesamt & 6.205 & 100,0 & 6.002 & 100,0 & 12.207 & 100,0 \\
\hline
\end{tabular}

Quelle: DEAS 2008 und 2014 ( $n=12.207)$, gewichtet, gerundete Angaben.

Tabelle 24-2 zeigt die demografische Zusammensetzung der Gruppen. Personen der zweiten Lebenshälfte mit Migrationshintergrund unterscheiden sich von denen ohne Migrationshintergrund und die einzelnen Migrantengruppen setzen sich unterschiedlich zusammen. Arbeitsmigrantinnen und -migranten und (Spät-)Aussiedlerinnen und -Aussiedler sind vergleichsweise älter als Personen ohne Migrationshintergrund, während andere Zugewanderte sowie Angehöri- 
ge der zweiten Generation deutlich jünger sind. Teilweise als Folge der unterschiedlichen Altersstruktur, vor allem aber begründet durch die jeweils unterschiedliche Migrationsgeschichte, unterscheiden sich die Gruppen deutlich in ihrer Aufenthaltsdauer in Deutschland: Mit Abstand am längsten leben Arbeitsmigrantinnen und -migranten in Deutschland (40,6 Jahre). Für (Spät-)
Aussiedlerinnen und -Aussiedler zeigt sich der bekannte Frauenüberschuss und unter den Arbeitsmigrantinnen und -migranten ein größerer Anteil von Männern (Schopf \& Naegele 2005). Migrantinnen und Migranten aus westlichen Ländern und der EU sind mehrheitlich Frauen, während das Geschlechterverhältnis in den verbleibenden Gruppen recht ausgeglichen ist.

Tabelle 24-2: Alters- und Geschlechterverteilung sowie Aufenthaltsdauer in Deutschland von Personen mit und ohne Migrationshintergrund, in den Jahren 2008 und 2014 (in Prozent)

\begin{tabular}{|c|c|c|c|c|c|}
\hline & \multicolumn{3}{|c|}{ Altersgruppe } & \multirow{2}{*}{$\begin{array}{l}\text { Aufent- } \\
\text { haltsdauer } \\
\text { (in Jahren) }\end{array}$} & \multirow{2}{*}{$\begin{array}{c}\text { Männer- } \\
\text { anteil }\end{array}$} \\
\hline & $\begin{array}{l}40-54 \\
\text { Jahre }\end{array}$ & $\begin{array}{l}55-69 \\
\text { Jahre }\end{array}$ & $\begin{array}{l}70-85 \\
\text { Jahre }\end{array}$ & & \\
\hline Ohne Migrationshintergrund & 41,4 & 33,5 & 25,1 & - & 48,4 \\
\hline Mit Migrationshintergrund & 51,4 & 33,3 & 15,4 & - & 47,5 \\
\hline ArbeitsmigrantInnen & 28,5 & 49,1 & 22,4 & 40,6 & 54,7 \\
\hline (Spät-)AussiedlerInnen & 42,7 & 33,6 & 23,7 & 24,6 & 42,4 \\
\hline $\begin{array}{l}\text { MigrantInnen aus westlichen und EU- } \\
\text { Ländern }\end{array}$ & 55,4 & 35,4 & 9,3 & 22,7 & 40,0 \\
\hline Sonstige MigrantInnen & 76,1 & 18,2 & 5,7 & 20,7 & 53,4 \\
\hline Zweite Generation & 51,7 & 35,8 & 12,5 & - & 50,9 \\
\hline
\end{tabular}

Quelle: DEAS 2008 und 2014 ( $n=12.133)$, gewichtet, gerundete Angaben.

Die zur Beschreibung der drei Dimensionen gesellschaftlicher Teilhabe herangezogenen Indikatoren wurden im Rahmen anderer Kapitel dieses Berichtsbandes entwickelt und verwendet.

Zur Abbildung der sozioökonomischen Lage werden die Armutsquote, der Anteil von Wohneigentümern sowie ein Indikator zur Schichtzugehörigkeit verwendet:

Armutsquote. Die Armutsquote basiert auf dem Äquivalenzeinkommen. Als arm gilt der Haushalt, dessen Einkommen unterhalb der 60-Prozent-Grenze des Medianeinkommens des jeweiligen Erhebungsjahres liegt (vgl. Kapitel 6).

Wohneigentum. Als Wohneigentümer werden diejenigen Befragten ausgewiesen, die angeben in einer Wohnung oder einem Haus zu leben, das ihnen selbst gehört (vgl. Kapitel 19).

Untere Sozialschicht. Diese Einstufung basiert auf einem Schichtindex, der die aktuelle oder die letzte berufliche Stellung zur Grundla- ge hat (in Anlehnung an Mayer \& Wagner 1999; vgl. auch Kapitel 7). Bei Partnerlosen wird die berufliche Stellung der Befragten verwendet. Bei Befragten, die sich aktuell in einer Partnerschaft befinden, ist die jeweils höchste berufliche Stellung maßgebend. Als untere Sozialschicht gelten un- und angelernte Arbeiter, Teilfacharbeiter sowie selbstständige Landwirte mit einer genutzten Fläche unter 19 Hektar.

Drei Facetten von Gesundheit werden abgebildet:

Funktionale Einschränkungen. Diese Zuordnung basiert auf der Sub-Skala der körperlichen Funktionsfähigkeit des SF36. Als körperlich eingeschränkt gelten Personen, die leichte bis starke funktionale Einschränkungen berichten, also einen Mittelwert auf der genannten Skala aufweisen, der größer als 1,3 ist (vgl. Kapitel 8).

Depressive Symptome. Depressivität wird anhand einer Allgemeinen Depressivitätsskala gemessen (Hautzinger \& Bailer 1993). Befragte 
haben demnach mindestens leichte depressive Symptome, wenn sie mehr als acht Punkte in dieser Skala aufweisen. Das trifft zu, wenn sie entweder für mehr als die Hälfte der Items angeben, unter den Symptomen mindestens „manchmal (ein bis zwei Tage lang)“ zu leiden oder wenn sie nur von einzelnen Symptomen, aber dafür häufiger betroffen sind (vgl. Kapitel 11).

Sportlich aktiv. Es wurde erhoben, wie häufig die Befragten verschiedene sportliche Aktivitäten wie Fußball spielen oder Schwimmen ausüben. Als aktiv werden Befragte eingestuft, die mindestens mehrmals wöchentlich Sport treiben (vgl. Kapitel 9).

Zur Abbildung der sozialen Einbindung und Partizipation finden folgende Indikatoren Verwendung:

Partnerschaft und Kinderzahl. Es wird betrachtet, ob der Befragte zum Zeitpunkt der Befragung in einer festen Partnerschaft lebt oder nicht. Zudem wird die Anzahl lebender Kinder berücksichtigt.

Intergenerationale Unterstützung. Um den Austausch zwischen Generationen zu beschreiben, wird zunächst die Ko-Residenz mit einem volljährigen Kind als besondere Form intergenerationaler Unterstützung berücksichtigt. Darüber hinaus werden die zwischen den Befragten und ihren außerhalb des Haushalts lebenden erwachsenen Kindern im Jahr vor der Befragung geleisteten Transfers von materieller (Geld-und Sachgeschenke) und instrumenteller Hilfe (Unterstützung im Haushalt) dargestellt (vgl. Kapitel 16).

Freizeitaktivitäten mit Freundinnen und Freunden. Für eine Reihe von Möglichkeiten der Freizeitgestaltung (wie spazieren gehen, Sport treiben, Gesellschaftsspiele) wird erfasst, wie viele davon mit Freundinnen oder Freunden erfolgen. Der Indikator reicht von (0) ,keine` bis (7) ,alle' (vgl. Kapitel 17).

Ehrenamt. Dieser Indikator gibt an, ob sich eine Person mindestens in einer der in einer Liste vorgegebenen Organisation oder Gruppen ehrenamtlich engagiert (vgl. Kapitel 5).

Analyse. Die im Folgenden berichteten Gruppenmittelwerte und prozentualen Anteile geben nicht lediglich die in der Stichprobe vorliegende Verteilung an. Vielmehr wurden die Werte im Rahmen multivariater Zusammenhangsanalysen geschätzt. Sie sind daher zum einen bezüglich Alter, Geschlecht und Landesteil an der Verteilung im Mikrozensus gewichtet. Zum anderen sind sie um Effekte, die sich aus der oben dargestellten gruppenspezifischen Verteilung nach Alter und Geschlecht ergeben, bereinigt. Die berichteten deskriptiven Werte beziehen sich daher jeweils auf eine hypothetische Person mittleren Alters, Geschlechts und Landesteils (vgl. Kapitel 2).

\subsection{Sozioökonomische Ausstattung}

Alle im Folgenden berichteten Ergebnisse bestätigen zunächst den vorliegenden Erkenntnisstand zur sozioökonomischen Ausstattung von Personen mit Migrationshintergrund in der zweiten Lebenshälfte - und das unter Kontrolle möglicher Einflüsse, die von gruppenspezifischer demografischer Zusammensetzung ausgehen können.

\section{Personen mit Migrationshintergrund sind sozioökonomisch schlechter gestellt als Personen ohne Migrationshintergrund.}

So zeigt sich für sie durchweg eine signifikante Schlechterstellung gegenüber den Personen ohne Migrationshintergrund. Abbildung 24-1 belegt, dass sie deutlich häufiger von Armut betroffen sind (26,0 Prozent gegenüber 9,7 Prozent), seltener über selbst bewohntes Wohneigentum verfügen (43,6 Prozent gegenüber 65,0 Prozent) und deutlich häufiger der sozialen Unterschicht angehören (16,4 Prozent gegenüber 3,8 Prozent). Dabei ist von starken Zusammenhängen zwischen diesen drei betrachteten Indikatoren auszugehen: So befördert eine niedrige berufliche Stellung eine materielle Schlechter- 
stellung, die wiederum die Chancen zum Erwerb von Wohneigentum herabsetzt. Darüber hinaus wird etwa ein Viertel des privaten Wohn- eigentums in Deutschland vererbt oder verschenkt (Wagner \& Mulder 2000) - eine Option, die Zugewanderten kaum zur Verfügung steht.

Abbildung 24-1: Anteile der Personen unterhalb der Armutsgrenze, von Zugehörigen der sozialen Unterschicht und von Wohneigentümern, in den Jahren 2008 und 2014 (in Prozent)

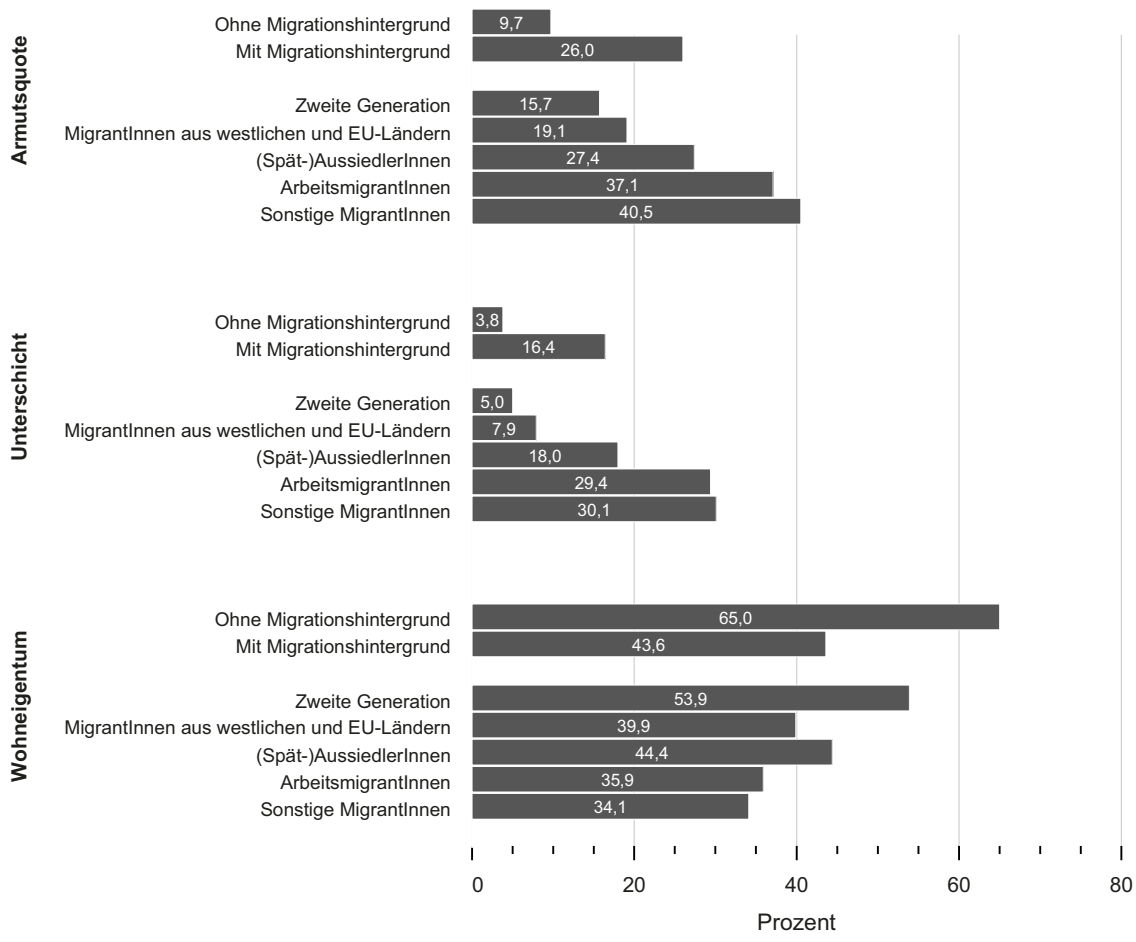

Quelle: DEAS 2008 und 2014; $(n=11.380$ für Armutsquote; $n=11.924$ für Unterschichtsquote; $n=12.129$ für Wohneigentum), gewichtet und kontrolliert nach Alter, Geschlecht, Landesteil. Gerundete Angaben; $(p<, 05)$.

Alle Gruppen unterscheiden sich signifikant von den Personen ohne Migrationshintergrund mit Ausnahme der zweiten Generation in der Kategorie Unterschicht.

\section{Die sozioökonomische Schlechterstellung betrifft vor allem frühere Arbeitsmigrantinnen und -migranten sowie Zugewanderte aus Ländern Afrikas, Asiens, Lateinamerikas, des Mittleren und Nahen Ostens sowie anderen Nicht-EU-Ländern.}

Bezogen auf die beiden großen Zuwanderergruppen bestätigt sich ein zum Teil deutlicher Unterschied zwischen Arbeitsmigrantinnen und -migranten einerseits und (Spät-)Aussiedlerinnen und -Aussiedlern andererseits. Dabei wird deutlich, dass letztere materiell besser aus- gestattet sind als erstere. Das Armutsrisiko von (Spät-)Aussiedlerinnen und -Aussiedlern ist geringer (27,4 Prozent) und sie verfügen zu einem größeren Anteil über Wohneigentum (44,4 Prozent) als frühere Arbeitsmigrantinnen und -migranten. Deren Armutsquote liegt bei 37,1 Prozent und sie bewohnen nur zu 35,9 Prozent Wohneigentum (unberücksichtigt hierbei ist mögliches Wohneigentum im Ausland). Diese materielle Diskrepanz dürfte nicht zuletzt der ungleichen Arbeitsmarktbeteiligung geschuldet sein. So sind (Spät-)Aussiedlerinnen und -Aussiedler im Durchschnitt besser gebildet und 
qualifiziert als frühere Arbeitsmigrantinnen und -migranten und gehören somit seltener der sozialen Unterschicht an (18,0 Prozent gegenüber 29,4 Prozent).

Die weitere systematische Ausdifferenzierung der Gruppe der Personen mit Migrationshintergrund deckt zusätzliche Unterschiede auf. So sind Zugewanderte aus westlichen Industrienationen und Ländern der EU, aber auch die Nachkommen der Zugewanderten am besten gestellt. Allerdings unterscheiden sich beide Gruppen in ihrer Ausstattung mit sozioökonomischen Ressourcen noch fast durchweg signifikant von der Bevölkerung ohne Migrationshintergrund.

Für die verbliebenen Migrantinnen und Migranten (aus Afrika, Asien, Lateinamerika, dem Mittleren und Nahen Osten sowie anderen Nicht-EU-Ländern) stellt sich die Situation am schlechtesten dar. Sie weisen durchweg die schlechtesten Quoten auf. Mehr als ein Drittel (40,5 Prozent) ist von Armut betroffen. 30,1 Prozent gehören zur Unterschicht, das heißt weisen eine niedrige berufliche Stellung auf und lediglich ein Drittel (34,1 Prozent) verfügt über Wohneigentum. Damit sind sie ähnlich benachteiligt wie die früheren Arbeitsmigrantinnen und -migranten. Die Situation der früheren Arbeitsmigrantinnen und -migranten verschärft sich allerdings in der zweiten Lebenshälfte noch dadurch, dass sich Risiken des Lebens zu kumulieren scheinen, wie die nachfolgende Betrachtung des Gesundheitszustandes nahelegt.

\subsection{Gesundheit}

Insgesamt unterscheiden sich Personen in der zweiten Lebenshälfte mit und ohne Migrationshintergrund signifikant auf allen betrachteten Dimensionen der Gesundheit: körperlich, psychisch und im Gesundheitsverhalten. Allerdings deckt der differenzierte Vergleich nach Gruppen auf, dass dieser Unterschied stark auf Benachteiligungen bei bestimmten Migrantengruppen zurückgeht.

\section{Die vergleichsweise schlechte Gesundheit von Personen mit Migrationshintergrund zeigt sich vor allem bei den Arbeitsmigrantinnen und -migranten.}

Nur die (Spät-)Aussiedlerinnen und -Aussiedler und mehr noch die Arbeitsmigrantinnen und -migranten weisen signifikant schlechtere Gesundheitswerte auf: So sind viele der Arbeitsmigrantinnen und -migranten in ihrer zweiten Lebenshälfte funktional eingeschränkt (38,1 Prozent), viele von ihnen zeigen depressi- ve Symptome (40,4 Prozent) und nur 16,3 Prozent sind mehrmals pro Woche sportlich aktiv. Vor allem die körperlichen Defizite mögen Ausdruck beziehungsweise Folge der gesundheitlich belastenden Arbeitsbedingungen sein, unter denen insbesondere die früheren Arbeitsmigrantinnen und -migranten erwerbstätig sind beziehungsweise waren. Nur etwas günstiger fallen die entsprechenden Werte für (Spät-)Aussiedlerinnen und -Aussiedler aus. Vor allem weisen sie ähnlich hohe Anteile depressiver Symptomatik auf. Es finden sich bei 35,8 Prozent depressive Symptome gegenüber 26,8 Prozent unter den Personen ohne Migrationshintergrund. Diese Unterschiede lassen sich nicht auf das durchschnittlich höhere Alter in diesen beiden Migrantengruppen zurückführen, da für das Alter (sowie Geschlecht und Landesteil), wie oben beschrieben, kontrolliert wurde. Es zeigt sich vielmehr, dass unter Konstanthaltung dieser demografischen Merkmale alle anderen Gruppen mit Migrationshintergrund der Bevölkerung ohne Migrationshintergrund viel stärker ähneln. 
Abbildung 24-2: Anteile der Personen mit funktionalen Einschränkungen, mit depressiven Symptomen,
und die mehrmals wöchentlich sportlich aktiv sind, in den Jahren 2008 und 2014 (in Prozent)

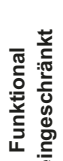

Zweite Generation

(Spät-)AussiedlerInnen

Arbeitsmigrantlnnen

Sonstige MigrantInnen

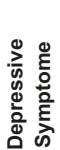

Ohne Migrationshintergrund Mit Migrationshintergrund

Zweite Generation MigrantInnen aus westlichen und EU-Ländern (Spät-)AussiedlerInnen ArbeitsmigrantInnen Sonstige MigrantInnen
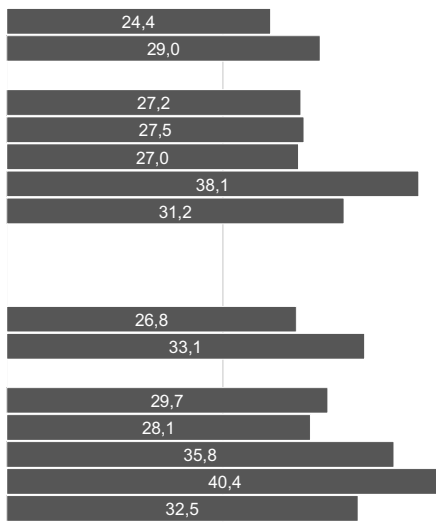

Ohne Migrationshintergrund Mit Migrationshintergrund

Zweite Generation MigrantInnen aus westlichen und EU-Ländern (Spät-)AussiedlerInnen ArbeitsmigrantInnen Sonstige MigrantInnen
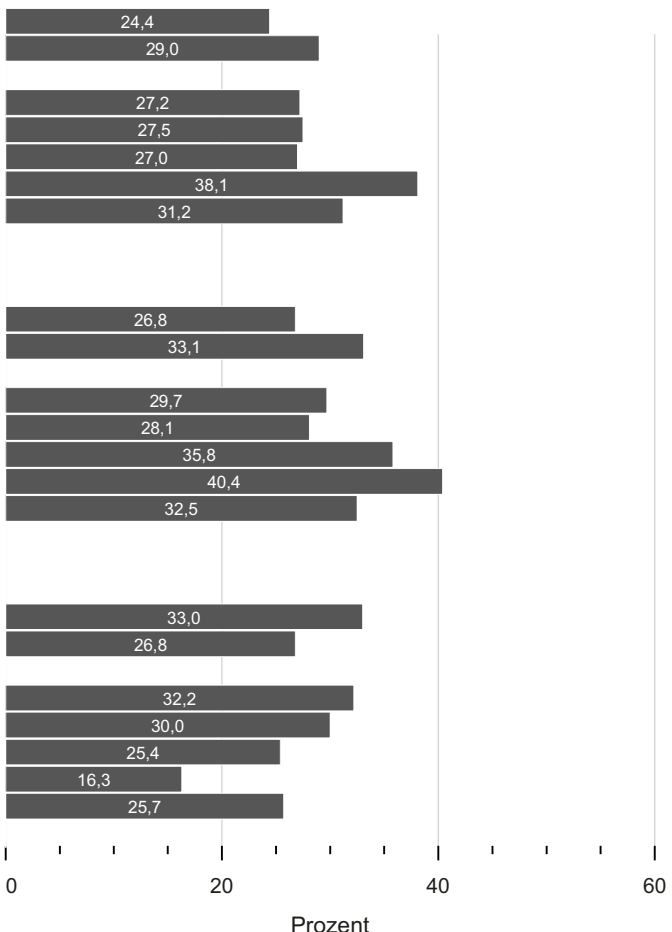

Quelle: DEAS 2008 und 2014; $n=12.127$ für funktionale Einschränkung; $n=11.863$ für depressive Symptome; $n=$ 12.128 für sportlich aktiv, gewichtet und kontrolliert nach Alter, Geschlecht, Landesteil. Gerundete Angaben; $(p<, 05)$. Funktionale Einschränkungen: Signifikante Unterschiede nur zwischen Personen ohne Migrationshintergrund und Personen mit Migrationshintergrund insgesamt sowie ArbeitsmigrantInnen. Depressive Symptome: Signfikante Unterschiede nur zwischen Personen ohne Migrationshintergrund und Personen mit Migrationshintergrund insgesamt, ArbeitsmigrantInnen sowie (Spät-)AussiedlerInnen. Sportlich aktiv: Signifikante Unterschiede nur zwischen Personen ohne Migrationshintergrund und Personen mit Migrationshintergrund insgesamt, ArbeitsmigrantInnen sowie (Spät-) AussiedlerInnen.

\begin{abstract}
Vor allem Männer mit Migrationshintergrund zeigen ein ungünstiges Gesundheitsverhalten und berichten eine schlechte Gesundheit.
\end{abstract}

Geschlechtsspezifische Betrachtungen belegen, dass der Gesundheitszustand von Männern schlechter ist als der von Frauen und sie ein ungünstigeres Gesundheitsverhalten zeigen. Dieses Muster findet sich in allen Gruppen (ohne Abbildung). 


\subsection{Soziale Einbindung und Partizipation}

Soziale Einbindung und Partizipation wird durch eine Reihe von sozialen Beziehungen gewährleistet. Mit zunehmendem Alter kommt dabei der Familie eine wachsende Bedeutung zu und hier vor allem den Beziehungen zwischen den Generationen. Generell ist der Anteil derer in der zweiten Lebenshälfte, die in einer Ehe oder Partnerschaft leben hoch: Das trifft auf 81,5 Prozent der Personen ohne und 79,7 Prozent der Personen mit Migrationshintergrund zu. Signifikante Unterschiede zwischen einzelnen Migrantengruppen finden sich nicht. Allerdings haben Personen mit Migrationshintergrund im Durchschnitt signifikant mehr Kinder $(2,1)$ als Ältere ohne Migrationshintergrund (1,8). Dabei ist die durchschnittliche Kinderzahl vor allem unter den Arbeitsmigrantinnen und -migranten $(2,2)$ und (Spät-)Aussiedlerinnen und -Aussiedlern $(2,1)$ sowie unter den sonstigen Migrantinnen und Migranten $(2,5)$ etwas höher - unter Kontrolle von Alters- und Geschlechterzusammensetzung (ohne Abbildung).

\section{Ko-Residenz mit erwachsenen Kindern ist unter Arbeitsmigrantinnen und -migranten und Zugewanderten aus Afrika, Asien, Lateinamerika, dem Mittleren und Nahen Osten sowie weiteren Nicht-EU-Ländern am weitesten verbreitet.}

Mit der Kinderzahl wachsen die Gelegenheiten für generationenübergreifendes Zusammenleben sowie für den Austausch von Unterstützung und Gütern. Abbildung 24-3 zeigt zunächst die Anteile derer in der zweiten Lebenshälfte, die mit erwachsenen Kindern zusammenleben. Deutliche Gruppenunterschiede sind zu erkennen: Während Ko-Residenz mit erwachsenen Kindern unter Angehörigen der zweiten Generation (20,5 Prozent) und Zuwanderinnen und Zuwanderern aus westlichen und EU-Ländern (21,1 Prozent) ähnlich gering verbreitet ist wie unter Personen ohne Migrationshintergrund (23,3 Prozent), wohnen Arbeitsmigrantinnen und -migranten (49,0 Prozent), (Spät-)Aussiedlerinnen und -Aus- siedler (30,0 Prozent) und sonstige Migrantinnen und Migranten (36,4 Prozent) signifikant häufiger mit einem erwachsenen Kind zusammen.

\section{Arbeitsmigrantinnen und -migranten, (Spät-)Aussiedlerinnen und -Aussiedler und Zugewanderte aus Afrika, Asien, Lateinamerika, dem Mittleren und Nahen Osten sowie weiteren Nicht-EU-Ländern unterstützen ihre erwachsenen Kinder am seltensten.}

Weiterhin finden sich in Abbildung 24-3 die Anteile der Personen, die mindestens eines ihrer außerhalb des Haushalts lebenden Kinder materiell oder instrumentell unterstützt haben oder von mindestens einem außerhäuslichen Kind diese Hilfen erhalten haben. Eine allgemeine Betrachtung der Austauschmuster zeigt zunächst: Während der Tausch von instrumentellen Hilfen zwischen den Generationen weitestgehend ausgeglichen ist, fällt das Verhältnis von Geben und Nehmen materieller Leistungen zu Gunsten der erwachsenen Kinder aus. Das heißt, im Durchschnitt erhalten mehr Kinder Geld- und Sachgeschenke von ihren Eltern, als diese umgekehrt von ihren Kindern erhalten. Dieses Muster zeigt sich grundsätzlich sowohl für Personen mit als auch ohne Migrationshintergrund.

Allerdings werden bei genauerer Betrachtung der gruppenspezifischen Unterstützungsleistungen interessante Abweichungen sichtbar. Diese betreffen vor allem materielle Transfers: Auch wenn diese Leistungen generell generationenabwärts fließen, werden sie von Eltern mit Migrationshintergrund seltener erbracht als von Eltern ohne Migrationshintergrund. Das gilt vor allem für die Gruppen der Arbeitsmigrantinnen und -migranten (16,7 Prozent), der (Spät-) Aussiedlerinnen und -Aussiedler (15,0 Prozent) sowie der sonstigen Migrantinnen und Migranten (16,1 Prozent). Dieses Muster ergänzt das Bild, das sich aus der beschriebenen materiellen Schlechterstellung sowie den hohen KoResidenzraten in diesen drei Gruppen ergibt. 
Eine gemeinsame Haushaltsführung von Eltern und erwachsenen Kindern kann einerseits als Antwort auf mangelnde Ressourcen zur Gründung eines eigenständiges Haushaltes verstanden werden und andererseits als eine besondere Form der intergenerationalen Hilfe. Hingegen finden sich in den Gruppen, in denen die Ko-Residenzraten niedrig sind, höhere Trans- ferquoten an Kinder. Eltern aus westlichen Industriestaaten und EU-Ländern (22,8 Prozent) sowie Angehörige der zweiten Generation (25,1 Prozent) unterstützen ihre erwachsenen Kinder materiell ähnlich häufig wie Eltern ohne Migrationshintergrund (25,3 Prozent). Informationen über die Höhe dieser Geldtransfers sind dabei nicht berücksichtigt.

\section{Abbildung 24-3: Anteile der Personen mit volljährigen ko-residenten Kindern sowie Unterstützungsraten zwischen volljährigen Kindern und ihren Eltern, in den Jahren 2008 und 2014 (in Prozent)}
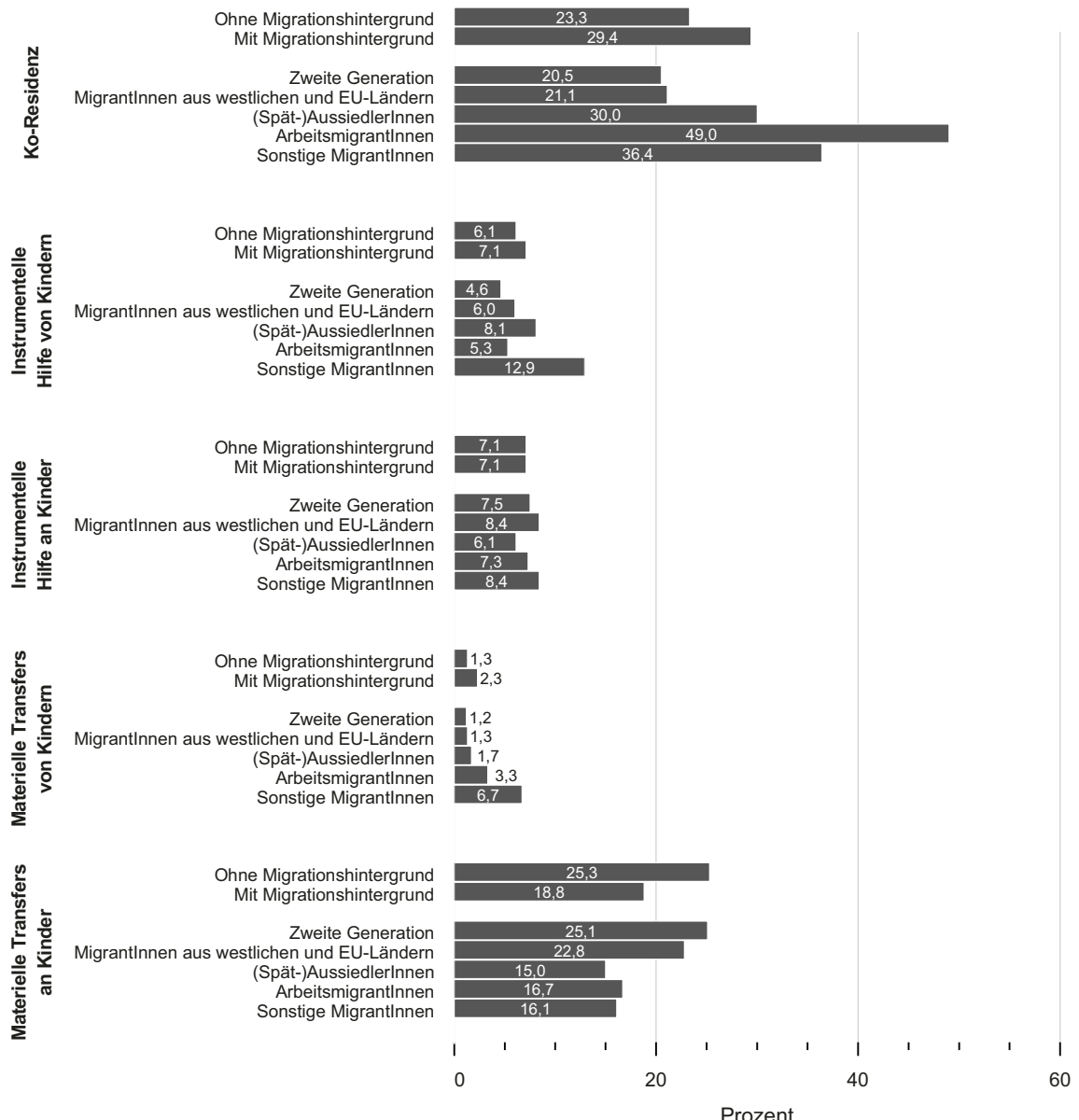

Quelle: DEAS 2008 und 2014; ( $n=11.790$ für Ko-Residenz; $8.116<=n<=8.229$ für Transferraten), gewichtet und kontrolliert nach Alter, Geschlecht, Landesteil. Gerundete Angaben; $(p<, 05)$.

Ko-Residenz: Signifikante Unterschiede nur zwischen Personen ohne Migrationshintergrund und ArbeitsmigrantInnen, (Spät-)AussiedlerInnen sowie sonstige MigrantInnen. Instrumentelle und materielle Transfers von Kindern: Signifikante Unterschiede nur zwischen Personen ohne Migrationshintergrund und sonstige MigrantInnen. Materielle Transfers an Kinder: Signifikante Unterschiede nur zwischen Personen ohne Migrationshintergrund und (Spät-)AussiedlerInnen. 
Zugewanderte aus Ländern Afrikas, Asiens, Lateinamerikas, des Mittleren und Nahen Ostens sowie anderen Nicht-EU-Ländern werden am häufigsten von ihren erwachsenen Kindern unterstützt.

Die sonstigen Migrantinnen und Migranten nehmen eine besondere Position ein, denn im Vergleich $\mathrm{zu}$ allen anderen Gruppen werden sie deutlich häufiger von ihren erwachsenen Kindern unterstützt. So erhalten 6,7 Prozent regelmäßige materielle Leistungen (gegenüber 1,3 Prozent bei den Eltern ohne Migrationshintergrund) und 12,9 Prozent werden instrumentell unterstützt (gegenüber 6,1 Prozent bei den Eltern ohne Migrationshintergrund). Damit weicht der intergenerationale Austausch in dieser Gruppe am stärksten von dem in Deutschland verbreiteten Muster ab, wonach die Bilanz von Geben und Nehmen weitgehend zugunsten der erwachsenen Kinder ausfällt. Die Bezie- hung zwischen erwachsenen Kindern und ihren Eltern ist hier am stärksten von gegenseitigen Verantwortlichkeiten und Abhängigkeiten geprägt - möglicherweise der schlechten und unsicheren Finanzlage in der Elterngeneration dieser Gruppe geschuldet.

\section{Migrantinnen und Migranten verbringen weniger Freizeit mit Freunden als Personen ohne Migrationshintergrund und Angehörige der zweiten Generation.}

Neben der Familie können Freunde und andere Sozialkontakte (zunehmend) bedeutsam für die soziale Einbindung sein. Die Abbildung 24-4 belegt, dass alle Personen mit Migrationshintergrund - abgesehen von den Angehörigen der zweiten Generation - ein durchweg signifikant geringeres Niveau aktiver Freizeitgestaltung mit Freunden aufweisen als Ältere ohne Migrationshintergrund.

Abbildung 24-4: Mittlere Anzahl von Freizeitaktivitäten mit Freunden, in den Jahren 2008 und 2014 (Mittelwert)

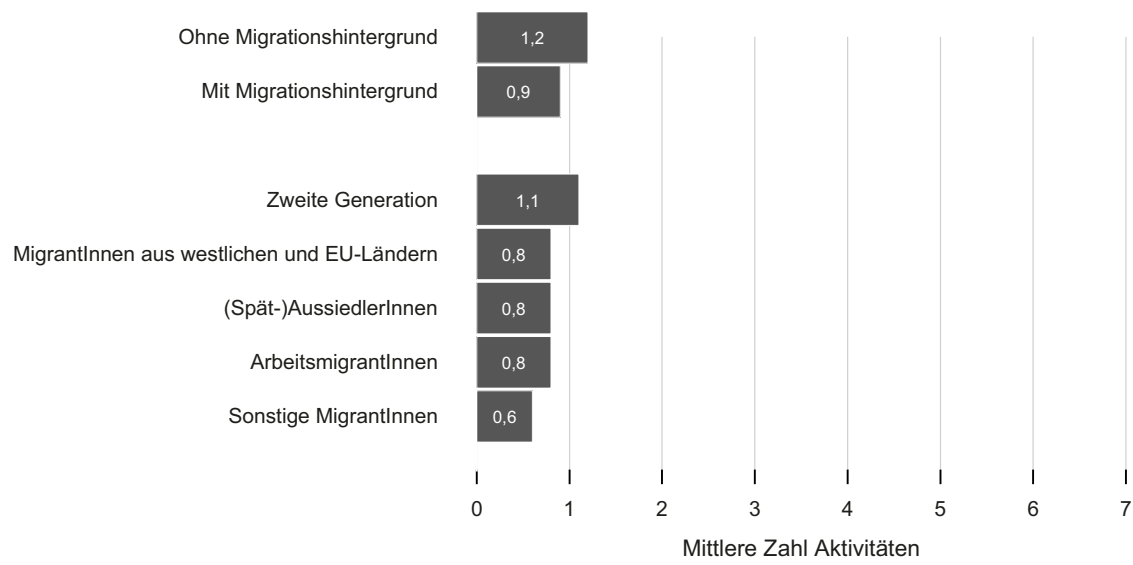

Quelle: DEAS 2008 und 2014; $(n=12.101)$, gewichtet und kontrolliert nach Alter, Geschlecht, Landesteil. Gerundete Angaben; $(p<, 05)$.

Alle Gruppen außer die zweite Generation unterscheiden sich signifikant von Personen ohne Migrationshintergrund.

Migrantinnen und Migranten üben seltener ein Ehrenamt aus als Personen ohne Migrationshintergrund und Angehörige der zweiten Generation.
Ein vergleichbares Muster zeigt sich für die Anteile ehrenamtlich Engagierter (Abbildung 24-5): Während sich 20,9 Prozent der Personen ohne Migrationshintergrund in diversen Orga- 
nisationen (wie Selbsthilfegruppen, Sportvereinen, kirchlichen oder religiösen Gruppen, Kulturvereinen oder Musikgruppen) ehrenamtlich betätigen, liegen die Anteile in allen Migrantengruppen deutlich unter zehn Prozent. Lediglich die Angehörigen der zweiten Generation engagieren sich mit 16,7 Prozent ähnlich häufig wie Personen ohne Migrationshintergrund. Diese Unterschiede sind nicht auf demografische Merkmale zurückzuführen - da die Befunde für
Geschlecht, Alter und Landesteil kontrolliert sind. Möglicherweise spielt aber die schlechtere gesundheitliche Lage sowie die nachteilige Qualifikations- und Erwerbssituation der Zugewanderten im Allgemeinen und der Arbeitsmigrantinnen und -migranten im Besonderen eine wichtige Rolle. So ist belegt, dass ehrenamtliches Engagement unter Erwerbstätigen und höher Gebildeten weiter verbreitet ist (vgl. Kapitel 5).

\section{Abbildung 24-5: Anteile der Personen, die ein Ehrenamt ausüben, in den Jahren 2008 und 2014 (in Prozent)}

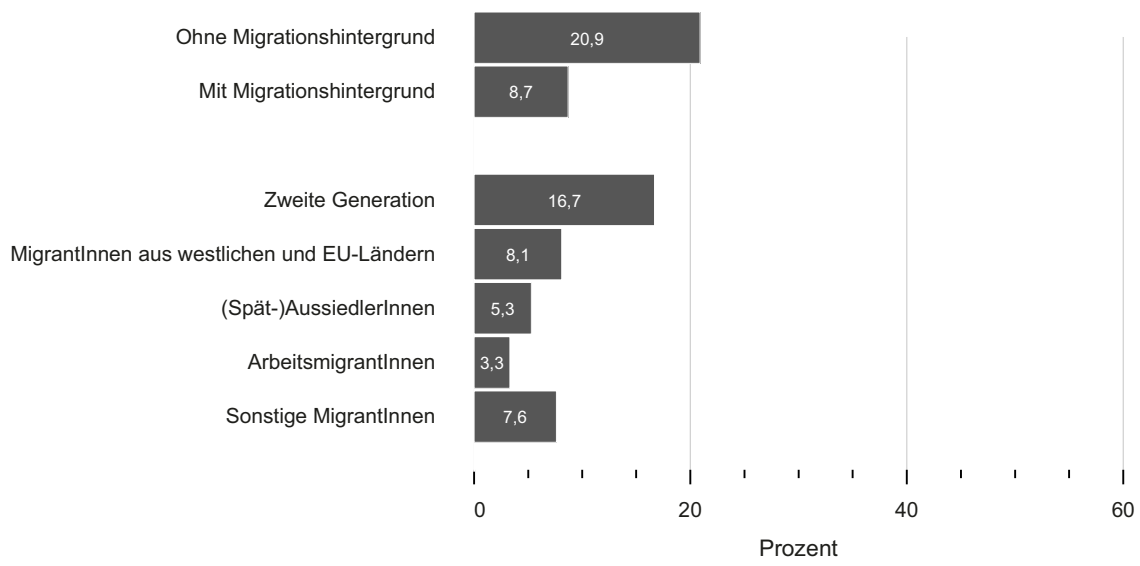

Quelle: DEAS 2008, 2014; $(n=12.116)$, gewichtet und kontrolliert nach Alter, Geschlecht, Landesteil. Gerundete Angaben; $(p<, 05)$.

Alle Gruppen außer die zweite Generation unterscheiden sich signifikant von Personen ohne Migrationshintergrund.

\subsection{Diskussion und Implikationen}

Die Daten des DEAS aus den Jahren 2008 und 2014 ermöglichen eine differenzierte Betrachtung der Lebenssituationen der Menschen mit Migrationshintergrund in der zweiten Lebenshälfte. Aufgrund der vergleichsweise jungen Einwanderungsgeschichte Deutschlands ist der große Teil von ihnen selbst im (jungen) Erwachsenenalter nach Deutschland eingewandert. Nicht $\mathrm{zu}$ vernachlässigen ist allerdings inzwischen auch der Anteil jener in der zweiten Lebenshälfte, die als Kinder von Zugewanderten nachgezogen sind oder in Einwandererfamilien in Deutschland geboren wurden. Sowohl anteilsmäßig als auch in absoluten Zahlen werden beide Gruppen in Deutschland in den kommenden Jahren weiter an Bedeutung gewinnen.

Mit der Frage nach den Lebensbedingungen und Teilhabechancen Älterer mit Migrationshintergrund orientiert sich das vorliegende Kapitel an der Diskussion um die Integration von Einwandererinnen und Einwanderern in Deutschland. Die Untersuchung der drei Dimensionen sozioökonomische Ausstattung, Gesundheit sowie soziale Einbindung und Partizipation - deckt 
wichtige Lebensbereiche ab, die zugleich die Voraussetzung für andere, darüber hinausgehende Formen der Teilhabe sind. Insgesamt bekräftigen die Befunde bisherige Forschungsergebnisse in eindrucksvoller Weise (Baykara-Krumme \& Hoff 2006; Schimany et al. 2012). Das ist insofern bedeutsam, als dass in der hier vorgestellten deskriptiven Analyse die zum Teil großen demografischen Differenzen zwischen den Gruppen herausgerechnet wurden. Entsprechend stellen die beobachteten Unterschiede keine demografischen Artefakte dar. So kann wiederholt und zuverlässiger als bisher geschlussfolgert werden: Personen in der zweiten Lebenshälfte, die einen Migrationshintergrund aufweisen, sind überdurchschnittlich häufig von Armut und sozialer Unterprivilegierung betroffen und berichten häufiger von gesundheitlichen Beeinträchtigungen. Die Einbindung in die Familie ist enger. Häufigere Ko-Residenz mit erwachsenen Kindern und mehr Unterstützung von ihnen sind Zeichen intensiver Generationensolidarität. Zugleich verweist dieser Befund auf die Bedarfe der Älteren, die diese Form der Unterstützung notwendig machen und umgekehrt die Möglichkeit von alternativen Ressourcentransfers an Kinder einschränken. Schließlich ist nicht zu vernachlässigen, dass die soziale Einbindung in außerfamiliale Kontexte in der Bevölkerung mit Migrationshintergrund etwas geringer ist. Außerdem engagieren sich Menschen mit Migrationshintergrund nach wie vor seltener ehrenamtlich.

Um der vielfach erwähnten Heterogenität der betrachteten Gruppe der Personen mit Migrationshintergrund gerecht zu werden, wurden in der vorliegenden Untersuchung verschiedene Migrantengruppen differenziert. Anders als in der Forschung zu Älteren mit Migrationshintergrund häufig üblich, stand dabei primär keine Untergliederung nach Herkunftsländern im Zentrum. Vielmehr erfolgte auf Basis der im DEAS verwendeten Indikatoren eine Differenzierung nach (rechtlich-historischem) Einreisekontext, Einwanderungsmotivation und Einwanderergeneration. Auf diese Weise können die damit verbundenen individuellen und gesellschaftlichen Bedingungen für Teilhabe und Integration angemessen berücksichtigt werden.
Tatsächlich zeigen sich in den Befunden zum Teil große Unterschiede, die die beachtliche Heterogenität der Gruppe der älteren Personen mit Migrationshintergrund hinsichtlich ihrer Partizipationsmöglichkeiten und -muster im Einwanderungsland Deutschland unterstreichen. So unterscheidet sich die Gruppe der Migrantinnen und Migranten aus den EU-Ländern und weiteren westlichen Ländern sowohl in ökonomischer, gesundheitlicher als auch sozialer Hinsicht nur wenig von den Personen ohne Migrationshintergrund.

Bisher neu sind die Befunde für die Angehörigen der zweiten Generation: Auch sie weisen größtenteils ähnliche Muster wie Ältere ohne Migrationshintergrund auf. Da nur wenig über die Herkunft und die Einreise- und Aufnahmebedingungen deren eingewanderter Eltern bekannt ist, können aus diesem Befund allerdings keine belastbaren Schlussfolgerungen $\mathrm{zu}$ den Ursachen der Ähnlichkeit gezogen werden. So deuten sich damit zwar bessere Integrationschancen in der zweiten Generation an sowie eine nachlassende Bedeutung der Benachteiligungen, die sich typischerweise aus der Migrationssituation für die Elterngeneration ergeben haben. Doch könnte bereits die Elterngeneration der aktuell Angehörigen der zweiten Generation in der zweiten Lebenshälfte selbst gut integriert (gewesen) sein, etwa weil sie mehrheitlich aus dem EU-Ausland zugewandert sind. Entsprechend ist Vorsicht geboten, aus diesem Befund voreilig einen allgemeingültigen, intergenerationalen Integrationsgewinn abzulesen.

Als besonders benachteiligt auf den hier untersuchten Dimensionen erweisen sich die $A r$ beitsmigrantinnen und -migranten sowie die außereuropäischen Migrantinnen und Migranten. Gegenüber der Bevölkerung ohne Migrationshintergrund sind sie vor allem materiell deutlich benachteiligt. Teilweise unterscheiden sie sich auch substanziell von den anderen Migrantengruppen. Zudem zeigen vor allem die Arbeitsmigrantinnen und -migranten ein nachteiliges Gesundheitsverhalten und haben die schlechteste Gesundheit. Besondere Aufmerksamkeit verlangt auch die Situation der Migrantinnen und Migranten aus den Ländern Afrikas, Asiens, Lateinamerikas, des Mittleren und Nahen 
Ostens sowie aus weiteren Nicht-EU-Ländern. Sowohl in materieller als auch in gesundheitlicher Hinsicht handelt es sich um eine besonders unterprivilegierte Gruppe, die allerdings - um die genauen Lebensumstände verstehen $\mathrm{zu}$ können - noch weiter ausdifferenziert werden müsste. Eine nicht zu vernachlässigende und in Zukunft noch bedeutsamer werdende Gruppe sind die Flüchtlinge, die als Ältere mit spezifischen Bedarfen und Ressourcen bisher kaum Aufmerksamkeit gefunden haben (Zeman \& Kalisch 2008).

Die Berücksichtigung von Personen in der zweiten Lebenshälfte mit Migrationshintergrund in den jüngsten DEAS-Erhebungen stellt einen großen Fortschritt dar sowie eine Aner- kennung der wachsenden Bedeutung dieser Bevölkerungsgruppe. Die vorgelegten Befunde leisten einen wertvollen Beitrag zur Darstellung der Lebenssituationen dieser vielschichtigen Personengruppe. Sie zeigen, dass die Integration von Personen mit Migrationshintergrund mit dem Ziel einer Angleichung der Lebensverhältnisse eine wichtige gesellschaftliche und politische Aufgabe ist und bleibt. Zugleich weisen sie auf spezifische Problemlagen hin und unterstreichen die Notwendigkeit einer differenziellen Betrachtung, um der Vielfalt der Lebenslagen gerecht $\mathrm{zu}$ werden und zugleich auf besonders benachteiligte Gruppen aufmerksam machen zu können.

\section{Literatur}

Baumann, J., \& Mika, T. (2012). Steigende Ungleichheit unter Aussiedlern und Spätaussiedlern im Alter. In: H. Baykara-Krumme, A. Motel-Klingebiel \& P. Schimany (Hrsg.) Viele Welten des Alterns. Ältere Migranten im alternden Deutschland (S. 127-157). Wiesbaden: Springer VS.

Baykara-Krumme, H. (2008a). Migrant families in Germany. Intergenerational solidarity in later life. Berlin: Weißensee Verlag.

Baykara-Krumme, H. (2008b). Reliable bonds? A comparative perspective of intergenerational support patterns among migrant families in Germany. In: C. Saraceno (Hrsg.) Families, Ageing and Social Policy. Intergenerational Solidarity in European Welfare States, 285-311. Cheltenham: Elgar.

Baykara-Krumme, H., \& Hoff, A. (2006). Die Lebenssituation älterer Ausländerinnen und Ausländer in Deutschland. In: C. Tesch-Roemer, H. Engstler \& S. Wurm (Hrsg.) Altwerden in Deutschland. Sozialer Wandel und individuelle Entwicklung in der zweiten Lebenshälfte (S. 447-518). Wiesbaden: VS Verlag für Sozialwissenschaften.

Baykara-Krumme, H., Klaus, D., \& Steinbach, A. (2011). Generationenbeziehungen in Deutschland. Ein Vergleich der Beziehungsqualität in einheimischen deutschen Familien, Familien mit türkischem Migrationshintergrund und Aussiedlerfamilien. In: J. Brüderl, L. Castiglioni \& N. Schumann (Hrsg.) Partnerschaft, Fertilität und intergenerationale Beziehungen. Ergebnisse

der ersten Welle des Beziehungs- und Familienpanels (S. 259-286). Würzburg: Ergon Verlag.

Baykara-Krumme, H., Motel-Klingebiel, A., \& Schimany, P. (Hrsg.) (2012). Viele Welten des Alterns: Ältere Migranten im alternden Deutschland. Wiesbaden: Springer VS.

Bundesministerium für Familie, Senioren, Frauen und Jugend (Hrsg.) (2005). Fünfter Bericht zur Lage der älteren Generation in der Bundesrepublik Deutschland - Potentiale des Alters in Wirtschaft und Gesellschaft. Der Beitrag älterer Menschen zum Zusammenhalt der Generationen. Berlin: Bundesministerium für Familie, Senioren, Frauen und Jugend.

Brzoska, P., \& Razum, O. (2015). Erreichbarkeit und Ergebnisqualität rehabilitativer Versorgung bei Menschen mit Migrationshintergrund. Bundesgesundheitsblatt - Gesundheitsforschung - Gesundheitsschutz, 58(6), 553-559.

Carnein, M., \& Baykara-Krumme, H. (2013). Einstellungen zur familialen Solidarität im Alter: Eine vergleichende Analyse mit türkischen Migranten und Deutschen. Zeitschrift für Familienforschung, 25(1), 29-52.

Deutscher Bundestag (Hrsg.) (2005). 6. Bericht über die Lage der Ausländerinnen und Ausländer in Deutschland. Berlin: Die Beauftragte der Bundesregierung für Migration, Flüchtlinge und Integration.

Dietzel-Papakyriakou, M. (2005). Potentiale älterer Migranten und Migrantinnen. Zeitschrift für Gerontologie und Geriatrie, 38(6), 396-406. 
Dietzel-Papakyriakou, M., \& Olbermann, E. (1996). Soziale Netzwerke älterer Migranten - Zur Relevanz familiärer und innerethnischer Unterstützung. Zeitschrift für Gerontologie und Geriatrie, 29(1), 34-41.

Frick, J. R., Grabka, M. M., Groh-Samberg, O., Hertel, F. R., Tucci, I., \& Fries, A. (2009). Alterssicherung von Personen mit Migrationshintergrund. Forschungsstudie. Endbericht zum Auftrag des BMAS, Projektgruppe "Soziale Sicherheit und Migration". Berlin: Bundesministerium für Arbeit und Soziales.

Greif, S., Grediga, G., \& Janikowski, A. (2003). Erwerbslosigkeit und beruflicher Abstieg von Aussiedlerinnen und Aussiedlern. In: K. J. Bade \& F. Oltmer (Hrsg.) Aussiedler: Deutsche Einwanderer aus Osteuropa (S. 81106). Göttingen: V \& R unipress.

Halm, D., \& Sauer, M. (2007). Bürgerschaftliches Engagement von Türkinnen und Türken in Deutschland. Wiesbaden: Springer VS.

Hautzinger, M., \& Bailer, M. (1993). Allgemeine Depressions-Skala (ADS). Manual. Weinheim: Beltz.

Heckmann, F. (2015). Integration von Migranten: Einwanderung und neue Nationenbildung. Wiesbaden: Springer VS.

Heinrich-Böll-Stiftung (Hrsg.). (2012). Altern in der Migrationsgesellschaft. Dossier. Berlin: Heinrich-BöllStiftung.

Hoffmann, E., \& Romeu Gordo, L. (2016). Lebenssituationen älterer Menschen mit Migrationshintergrund. In: Statistisches Bundesamt \& Wissenschaftszentrum Berlin für Sozialforschung (Hrsg.) Datenreport 2015 (S. 64-73). Berlin: Bundeszentrale für politische Bildung.

Höhne, A., \& Schubert, M. (2007). Vom Healthy-migrant-Effekt zur gesundheitsbedingten Frühberentung. Erwerbsminderungsrenten bei Migranten in Deutschland. In: Deutsche Rentenversicherung Bund (Hrsg.) Etablierung und Weiterentwicklung. Bericht vom vierten Workshop des Forschungsdatenzentrums der Rentenversicherung (FDZ-RV) vom 28. bis 29. Juni in Berlin. Bd. 55/2007. Bad Homburg, (S. 103-125). Berlin: Deutsche Rentenversicherung Bund.

Hubert, S., Althammer, J., \& Korucu-Rieger, C. (2009). Soziodemographische Merkmale und psychophysisches Befinden älterer türkischer Migrantinnen und Migranten in Deutschland: eine Untersuchung auf Basis der Haupt-und Zusatzbefragung des Generations and Gender Survey der ersten Welle. Berlin: Pro Business.

Huth, S. (2012). Bürgerschaftliches Engagement von älteren MigrantInnen. In: Heinrich-Böll-Stiftung (Hrsg.) Altern in der Migrationsgesellschaft. Dossier (S. 27-31). Berlin: Heinrich-Böll-Stiftung.

Kohls, M. (2012). Leben ältere Migranten länger? In: H. Baykara-Krumme, A. Motel-Klingebiel \& P. Schima- ny (Hrsg.) Viele Welten des Alterns. Ältere Migranten im alternden Deutschland (S. 201-222). Wiesbaden: Springer VS.

Kunst, A. E., Bos, V., Lahelma, E., Bartley, M., Lissau, I., Regidor, E., Mielck, A., Cardano, M., Dalstra, J. A. A., Geurts, J. J. M., Helmert, U., Lennartsson, C., Ramm, J., Spadea, T., Stronegger, W. J., \& Mackenbach, J. P. (2005). Trends in socioeconomic inequalities in selfassessed health in 10 European countries. International Journal of Epidemiology, 34(2), 295-305.

Mayer, K.-U., \& Wagner, M. (1999). Lebenslagen und soziale Ungleichheit im hohen Alter. In: U. Lindenberger, J. Smith, K.-U. Mayer \& P. B. Baltes (Hrsg.) Die Berliner Altersstudie (S. 251-275). Berlin: Akademie Verlag.

Meier-Braun, K.-H. (2002). Deutschland, Einwanderungsland. Berlin: Suhrkamp.

Menning, S., \& Hoffmann, E. (2009). Ältere Migrantinnen und Migranten [Report Altersdaten 01/2009]. Berlin: Deutsches Zentrum für Altersfragen.

Mika, T., \& Tucci, I. (2006). Alterseinkommen bei Zuwanderern. Gesetzliche Rente und Haushaltseinkommen bei Aussiedlern und Zuwanderern aus der Türkei und dem ehemaligen Jugoslawien im Vergleich zur deutschen Bevölkerung. Berlin: Deutsches Institut für Wirtschaftsforschung.

Olbermann, E. (2012). Gesundheitliche Situation und soziale Netzwerke älterer MigrantInnen. In: HeinrichBöll-Stiftung (Hrsg.) Altern in der Migrationsgesellschaft. Dossier (S. 33-37). Berlin: Heinrich-Böll-Stiftung.

Özcan, V., \& Seifert, W. (2006). Lebenslage älterer Migrantinnen und Migranten in Deutschland. Berlin: Deutsches Zentrum für Altersfragen.

Romeu Gordo, L. (2012). Erwerbsverläufe älterer MigrantInnen. In: Heinrich-Böll-Stiftung (Hrsg.) Altern in der Migrationsgesellschaft. Dossier (S. 18-21). Berlin: Heinrich-Böll-Stiftung.

Sahyazici, F., \& Huxhold, O. (2012). Depressive Symptome bei älteren türkischen Migrantinnen und Migranten. In: H. Baykara-Krumme, A. Motel-Klingebiel \& P. Schimany (Hrsg.) Viele Welten des Alterns. Ältere Migranten im alternden Deutschland (S. 181-200). Wiesbaden: Springer VS.

Schimany, P., \& Baykara-Krumme, H. (2012). Zur Geschichte und demografischen Bedeutung älterer Migrantinnen und Migranten in Deutschland. In: H. Baykara-Krumme, A. Motel-Klingebiel \& P. Schimany (Hrsg.) Viele Welten des Alterns. Ältere Migranten im alternden Deutschland (S. 43-73). Wiesbaden: Springer VS.

Schimany, P., Rühl, S., \& Kohls, M. (Hrsg.). (2012). Ältere Migrantinnen und Migranten. Entwicklungen, Lebenslagen, Perspektiven. Nürnberg: Bundesamt für Migration und Flüchtlinge. 
Schopf, C., \& Naegele, G. (2005). Alter und Migration. Zeitschrift für Gerontologie und Geriatrie, 38(6), 384-395.

Spallek, J., \& Razum, O. (2008). Erklärungsmodelle für die gesundheitliche Situation von Migrantinnen und Migranten. In: U. Bauer, U. H. Bittlingmayer \& M. Richter (Hrsg.) Health Inequalities. Determinanten und Mechanismen gesundheitlicher Ungleichheit (S. 271-288). Wiesbaden: VS Verlag für Sozialwissenschaften.

Statistisches Bundesamt. (2015). Mikrozensus 2013 Sonderauswertungen für das Deutsche Zentrum für Altersfragen. Wiesbaden: Statistisches Bundesamt.

Steinbach, A. (2013). Family structure and parent-child contact: A comparison of native and migrant families. Journal of Marriage and Family, 75(5), 1114-1129.

Thum, M., Delkic, E., Kemnitz, A., Kluge, J., Marquardt, G., Motzek, T., Nagl, W., \& Zwerschke, P. (2015). Auswirkungen des demografischen Wandels im Einwanderungsland Deutschland. Bonn: Friedrich-Ebert-Stiftung.

Tucci, I. (2012). Die Einkommens-und Wohnsituation älterer MigrantInnen. In: Heinrich-Böll-Stiftung (Hrsg.) Altern in der Migrationsgesellschaft. Dossier (S. 12-17). Berlin: Heinrich-Böll-Stiftung.
Tucci, I., \& Yıldız, S. (2012). Das Alterseinkommen von Migrantinnen und Migranten: zur Erklärungskraft von Bildungs- und Erwerbsbiografien. In: H. BaykaraKrumme, A. Motel-Klingebiel \& P. Schimany (Hrsg.) Viele Welten des Alterns. Ältere Migranten im alternden Deutschland (S. 101-126). Wiesbaden: Springer VS.

Vogel, C. (2012). Generationenbeziehungen der (Spät-) Aussiedler. Forschungsstand und exemplarische Befunde zu Einstellungen in Bezug auf familiale Unterstützungsleistungen. In: H. Baykara-Krumme, A. Motel-Klingebiel \& P. Schimany (Hrsg.) Viele Welten des Alterns. Ältere Migranten im alternden Deutschland (S. 289-313). Wiesbaden: Springer VS.

Wagner, M., \& Mulder, C. H. (2000). Wohneigentum im Lebenslauf: Kohortendynamik, Familiengründung und sozioökonomische Ressourcen. Zeitschrift für Soziologie 29(1), 44-59.

Zeman, P. (2005). Ältere Migranten in Deutschland. Befunde zur soziodemographischen, sozioökonomischen und psychosozialen Lage sowie zielgruppenbezogene Fragen der Politik- und Praxisfeldentwicklung. Berlin: Deutsches Zentrum für Altersfragen.

Zeman, P., \& Kalisch, D. (2008). Die Situation älterer Flüchtlinge. Belastungen und Potenziale. Informationsdienst Altersfragen, 35(4), 2-7.

Open Access Dieses Kapitel wird unter der Creative Commons Namensnennung 2.5 International Lizenz (http://creativecommons.org/licenses/by/2.5/deed.de) veröffentlicht, welche die Nutzung, Vervielfältigung, Bearbeitung, Verbreitung und Wiedergabe in jeglichem Medium und Format erlaubt, sofern Sie den/die ursprünglichen Autor(en) und die Quelle ordnungsgemäß nennen, einen Link zur Creative Commons Lizenz beifügen und angeben, ob Änderungen vorgenommen wurden.

Die in diesem Kapitel enthaltenen Bilder und sonstiges Drittmaterial unterliegen ebenfalls der genannten Creative Commons Lizenz, sofern sich aus der Abbildungslegende nichts anderes ergibt. Sofern das betreffende Material nicht unter der genannten Creative Commons Lizenz steht und die betreffende Handlung nicht nach gesetzlichen Vorschriften erlaubt ist, ist für die oben aufgeführten Weiterverwendungen des Materials die Einwilligung des jeweiligen Rechteinhabers einzuholen. 